\title{
Mean-square exponential input-to-state stability of delayed Cohen-Grossberg neural networks with Markovian switching based on vector Lyapunov functions
}

\author{
Zhihong $\mathrm{Li}^{a}$, Lei Liu ${ }^{a, *}$, Quanxin Zhu ${ }^{b, c}$ \\ ${ }^{a}$ College of Science, Hohai University, Nanjing, 210098, China \\ ${ }^{b}$ School of Mathematical Sciences and Institute of Finance and Statistics, Nanjing Normal University, Nanjing, 210023, China \\ ${ }^{c}$ Department of Mathematics, University of Bielefeld, Bielefeld D-33615, Germany.
}

\begin{abstract}
This paper studies the mean-square exponential input-to-state stability of delayed Cohen-Grossberg neural networks with Markovian switching. By using the vector Lyapunov function and property of M-matrix, two generalized Halanay inequalities are established. By means of the generalized Halanay inequalities, sufficient conditions are also obtained, which can ensure the exponential input-to-state stability of delayed Cohen-Grossberg neural networks with Markovian switching. Two numerical examples are given to illustrate the efficiency of the derived results.
\end{abstract}

Keywords: Exponential input-to-state stability, Cohen-Grossberg neural network, Halanay inequality, M-matrix, Time delay, Markovian switching

\section{Introduction}

In 1983, Cohen and Grossberg presented a neural network [1], which is now called Cohen-Grossberg neural network(CGNN). As we know, Cohen-Grossberg neural networks include the famous Hopfield neural networks, cellular neural networks as its special cases. This model has been paid much considerable attention due to its wide applications in various areas such as pattern classification, associative memory, parallel computation, optimization, system identification and control, moving object speed detection and so on. Accordingly, a great number of results have been published concerning CGNNs [2]-[7] and the references cited therein.

Time delays are unavoidable in hardware implementation owing to the finite switching speed of amplifiers. It may often leads to the oscillation, divergence, and even instability during the application of neural networks. In the past few years, delayed neural networks have been extensively studied by researchers and there have appeared a large number of results in the literature, for instance [2], [5], [7]-[9] and references therein. In addition, since Markovian jump linear systems were firstly introduced in early 1960s, various systems driven by continuous time Markovian chains have been widely employed to practical systems where they may experience abrupt changes in system structure and parameters. In such a case, neural networks can be represented by a switching model which can be regarded as a set of parametric configurations switching from one to another according to a given Markovian chain (see [10]-[15]). Taking the time delays and Markovian switching into account, it is actually valuable to investigate the stability of delayed Cohen-Grossberg neural networks (DCGNNs) with Markovian switching.

It is well-known that the stability of neural network is not only the most basic and important problem but also the foundation of neural network's applications. Recently, there have been a lot of literature on the stability analysis of neural networks reported in the literature (see [16]-[22]). On the other hand, the input-to-state stability (ISS) is one of the useful properties for nonlinear systems (see [5], [7]). The ISS concept means that no matter what the initial state is, if the inputs are uniformly small, then the state of the neural networks must eventually be small. It offers an effective way to handle the stabilization of neural network applications in the presence of various uncertainties.

\footnotetext{
* Corresponding Author.

E-mail addresses: liulei_hust@hhu.edu.cn(L. Liu)
} 
Recently, some results on the ISS properties are obtained for neural networks. For example, Sanchez and Perez [23] investigated the ISS properties and gave some matrix norm conditions on ISS of recurrent neural networks (RNNs) firstly. Ahn utilized Lyapunov function method to discuss robust stability problem for a class of RNNs, and also some LMI sufficient conditions have been proposed to guarantee the ISS in [24]. It is Zhu and Cao who firstly investigated the exponential ISS for stochastic neural networks in [7] and [25]. Recently, we have also noticed that some scholars begin to discuss the exponential stability of neural networks by means of the vector Lyapunov function methods, see e.g. [5], [8], [12]. For example, Shen and Wang [12] have considered the exponential stability of DRNNs with Markovian switching by a generalized vector Halanay inequality. By using the Razuminkhin technique, two sufficient criteria on mean square exponential ISS of stochastic delayed exponential ISS of are derived in [5]. It is well-known that the existing criteria can not handle the exponential ISS of DCGNNs with Markovian switching, since the simulataneous presence of external input item and switching mechanism. To the best of our knowledge, the exponential ISS of DCGNNs with Markovian switching has scarcely been investigated. As such, this issue constitutes the first motivation of this paper.

Moreover, for most of the existing stability criteria for neural networks, described by scalar Lyapunov function or vector Lyapunov function, the time-delayed item and the non-delay item are separated in corresponding $\mathscr{L}$-operator differential inequality. It is well-known that the cross items will inevitably arise when using the Lyapunov function to study the stability of delayed systems. While most scholars choose to use the elemental inequality to deal with the cross items. As a result, these proposed methods seem to be more conservative. Naturally, an interesting question is generated whether the cross items could arise in the vector $\mathscr{L}$-operator differential inequality? Solving this problems is the second motivation of this paper.

Summarizing the above statements, the focus of this paper is to discuss the exponential ISS for DCGNNs with Markovian switching, and obtain the criteria described by vector $\mathscr{L}$-operator differential inequality with cross items. The main contributions of this paper lie in two aspects: 1) By using the vector Lyapunov functions and stochastic analysis technique, two generalized stochastic vector Halanay inequalities are established; 2) Based on the novel Halanay inequalities, sufficient algebraic criteria with less conservative are obtained to ensure the ISS in mean square sense.

The remainder of this paper is organized as follows. Section 2 introduces the model of DCGNNs with Markovian switching and gives some necessary notations. Section 3 presents the main results. Two numerical examples are given to show the effectiveness of the main results in Section 4. Finally, concluding remarks are made in Section 5.

\section{Models and preliminaries}

Throughout this paper, unless otherwise specified, we let $\left(\Omega, \mathcal{F},\left\{\mathcal{F}_{t}\right\}_{t \geq 0}, \mathcal{P}\right)$ be a complete probability space with a filtration $\left\{\mathcal{F}_{t}\right\}_{t \geq 0}$ satisfying the usual conditions (i.e., it is right continuous and $\mathcal{F}$ contains all P-null sets). Let $\tau>0$ and $C=C\left([-\tau, 0], R^{n}\right)$ denote the family of continuous functions $\varphi$ from $[-\tau, 0]$ to $R^{n}$ with the norm $\|\varphi\|_{\tau}=\sup _{-\tau \leq \theta \leq 0}|\varphi(\theta)|$, where $|\cdot|$ is the Euclidean norm in $R^{n}$. Denote by $\mathcal{L}_{\mathscr{F}_{t}}^{2}$ the family of all $C\left([-\tau, 0] ; R^{n}\right)$-valued, $\mathcal{F}_{t}-$ adapted stochastic variables $\phi=\{\phi(s),-\tau \leq s \leq 0\}$ such that $\int_{-\tau}^{0} E|\phi(s)|^{2} d s<\infty$, where $E$ stands for the correspondent expectation operator with respect to the given probability measurable $\mathcal{P}$. The set of all essentially bounded functions $u: R_{+} \rightarrow R^{n}$, endowed with essential supremum norm $\|u\|_{\infty}=\sup \{|u(t)|, t \geq 0\}$, is denoted by $L_{\infty}^{n}$. A function $\varphi: R_{+} \rightarrow R_{+}$is said to be class of $\mathcal{K}$ if it is continuous and strictly increasing and satisfies $\varphi(0)=0$; it is of class $\mathcal{K}_{\infty}$ if in addition $\varphi(s) \rightarrow \infty$ as $s \rightarrow \infty$. Let $G$ is a vector or matrix. By $G \geq 0$ we mean that each element of $G$ is non-negative. By $G \gg 0$ we mean that all elements of $G$ are positive. And if $G=\left(g_{i j}\right)_{n \times n} \in R^{n \times n}$, we denote $|G|=\left(\left|g_{i j}\right|\right)_{n \times n}, \bar{G}=\left(\bar{g}_{i j}\right)_{n \times n}$ with $\bar{g}_{i j}=g_{i j}(i \neq j), \bar{g}_{i i}=g_{i i}^{+}=\max \left\{g_{i i}, 0\right\}, i=1, \cdots, n$. Moreover, we also adopt here the traditional notation by letting

$$
Z^{\mathrm{n} \times \mathrm{n}}=\left\{A=\left\{a_{i j}\right\}_{\mathrm{n} \times \mathrm{n}}: a_{i j} \leq 0, i \neq j\right\} .
$$

Let $\{r(t)(t \geq 0)\}$ be a right-continuous Markovian chain on the probability space taking values in a finite state space $\mathcal{M}=\{1,2, \cdots, N\}$ with generator $\Gamma=\left(\gamma_{i j}\right)_{N \times N}$ given by

$$
P\{r(t+\Delta)=j \mid r(t)=i\}= \begin{cases}\gamma_{i j} \Delta+o(\Delta) & i \neq j \\ 1+\gamma_{i i} \Delta+o(\Delta) & i=j,\end{cases}
$$


where $\Delta>0$. Here $\gamma_{i j} \geq 0$ is the transition rate from $i$ to $j$ if $i \neq j$ while $\gamma_{i i}=-\Sigma_{i \neq j} \gamma_{i j}$.

In this paper, we consider a DCGNN with Markovian switching

$$
\begin{aligned}
d x_{k}(t)= & h_{k}\left(x_{k}(t)\right)\left[-d_{k}\left(r(t), x_{k}(t)\right)+\sum_{l=1}^{n} a_{k l}(r(t)) f_{l}\left(x_{l}(t)\right)\right. \\
& \left.+\sum_{l=1}^{n} b_{k l}(r(t)) f_{l}\left(x_{l}\left(t-\tau_{l}(t)\right)\right)+u_{k}(t)\right] d t, \quad k=1,2, \cdots, n,
\end{aligned}
$$

for $t \geq 0$ with initial value $\xi \in C, r_{0} \in \mathcal{M}$, where $x(t)=\left(x_{1}(t), x_{2}(t), \cdots, x_{n}(t)\right)^{T}$ is the state of the neuron at time $t, H(x(t))=\operatorname{diag}\left(h_{1}\left(x_{1}(t)\right), h_{2}\left(x_{2}(t)\right), \cdots, h_{n}\left(x_{n}(t)\right)\right)$ represents the amplification function of the neuron at time $t$, $D(i, x(t))=\operatorname{diag}\left(d_{1}\left(i, x_{1}(t)\right), d_{2}\left(i, x_{2}(t)\right), \cdots, d_{n}\left(i, x_{n}(t)\right)\right)^{T}$ is the appropriately behaved function dependent on $t$ and on the state processes $x(t)$, while $A(i)=\left(a_{k l}(i)\right)_{n \times n}$ and $B(i)=\left(b_{k l}(i)\right)_{n \times n}$ describe the connection weight matrices associated without delays and with delays, respectively. $\tau_{l}(\cdot) l=1, \cdots, n$ denote the time-varying delay, that satisfies $0 \leq$ $\tau_{l}(t) \leq \tau$, where $\tau$ is the maximal delay. $U(t)=\left(u_{1}(t), u_{2}(t), \cdots, u_{n}(t)\right)^{T}$ is the external input function at time $t$, $f(x(t))=\left(f_{1}\left(x_{1}(t), f_{2}\left(x_{2}(t), \cdots, f_{n}\left(x_{n}(t)\right)^{T}\right.\right.\right.$ and $f(x(t-\tau(t)))=\left(f_{1}\left(x_{1}\left(t-\tau_{1}(t)\right), f_{2}\left(x_{2}\left(t-\tau_{2}(t)\right), \cdots, f_{n}\left(x_{n}\left(t-\tau_{n}(t)\right)\right)^{T}\right.\right.\right.$ are vector-valued activation functions.

In order to prove our main results, we make the following assumptions on the amplification functions, behaved functions, and activation functions.

Assumption 1: There exist positive constants $\underline{h}_{k}, \bar{h}_{k}$ such that

$$
0<\underline{h}_{k} \leq h_{k}(x) \leq \bar{h}_{k}, x \in R, k=1,2, \cdots, n .
$$

Assumption 2: For $i \in \mathcal{M}$, there exist positive constants $\delta_{k}(i)$ such that

$$
x d_{k}(i, x) \geq \delta_{k}(i) x^{2}, x \in R, k=1,2, \cdots, n .
$$

Assumption 3:. There exist positive constants $M_{k}$ such that

$$
0 \leq \frac{f_{k}(x)-f_{k}(y)}{x-y} \leq M_{k}, x, y \in R, k=1,2, \cdots, n .
$$

Let $C^{2,1}\left(R^{n} \times R_{+} \times \mathcal{M} ; R_{+}\right)$denote the family of all nonnegative functions $V(x, t, i)$ on $R^{n} \times R_{+} \times \mathcal{M}$, which are continuously twice differentiable in $x$ and once in $t$. Then we can define an operator $\mathscr{L} V$ from $R^{2 n} \times R_{+} \times \mathcal{M}$ to $R$ by

$$
\mathscr{L} V(x, y, t, i)=V_{t}(x, t, i)+V_{x}(x, t, i)\{H(x)[-D(i, x)+A(i) f(x)+B(i) f(y)+U(t)]\}+\sum_{j=1}^{n} \gamma_{i j} V(x, t, j) .
$$

Definition 1: The DCGNN with Markovian switching (1) is said to be mean-square exponentially input-to-state stable, if there exist constants $\lambda>0, L>0$ and $\varphi \in \mathcal{K}_{\infty}$ such that the solution $x\left(t ; \xi, r_{0}\right)$ satisfies

$$
E\left|x\left(t ; \xi, r_{0}\right)\right|^{2} \leq L E\|\xi\|_{\tau}^{2} e^{-\lambda t}+\varphi\left(\|u\|_{\infty}^{2}\right) .
$$

for any $\xi \in \mathcal{L}_{\mathcal{F}_{t}}^{2}, u(t) \in L_{\infty}^{n}, r_{0} \in \mathcal{M}$ and $t \geq 0$.

Definition 2: Let $g: R \rightarrow R$ be a continuous function, define $D^{+} g$ the upper right Dini-derivative of $g$ as

$$
D^{+} g(t)=\limsup _{\delta \rightarrow 0^{+}} \frac{g(t+\delta)-g(t)}{\delta} .
$$

Lemma 2.1: (Berman and Plemmons, 1979 [28]). If $A \in Z^{\mathrm{n} \times \mathrm{n}}$, then the following statements are equivalent:

(1) $A$ is a nonsingular M-matrix.

(2) $A$ is semipositive; that is, there exists $x \gg 0$ in $R^{n}$ such that $A x \gg 0$.

(3) $A^{-1}$ exists and its elements are all nonnegative.

(4) All the leading principal minors of $A$ are positive.

Remark 2.1: It is clear that mean-square exponential ISS is mean-square exponential stability when $U \equiv 0$. Therefore, mean-square exponential ISS is more general than mean-square exponential stability. 


\section{Main results}

In this section, the stability is studied for the DCGNN with Markovian switching (1). To begin with, we introduce the following Lemmas which will be used in the proof of the main results.

\subsection{Stochastic vector Halanay inequality}

Lemma 3.1: Assume that $x(t)$ is a real-valued $\mathcal{F}_{t}$-adapted process on $[-\tau, \infty)$, let $P=\operatorname{diag}\left\{p_{1}, \cdots, p_{n}\right\}, Q=\left(q_{k l}\right)_{n \times n}$ with $p_{k}>0, q_{k l} \geq 0, k, l=1,2, \cdots, n$, and $I_{1}(t), \cdots, I_{n}(t) \in L_{\infty}^{n}$. Moreover, there exist functions $V_{1}, \cdots, V_{n} \in$ $C\left(R^{n} \times R_{+} \times \mathcal{M}, R_{+}\right)$such that

$$
\begin{aligned}
D^{+} E V_{k}(x(t), t, r(t)) \leq & -p_{k} E V_{k}(x(t), t, r(t))+\sum_{l=1}^{n} q_{k l} \sup _{-\tau \leq \theta \leq 0} \sqrt{E V_{l}(x(t+\theta), t+\theta, r(t+\theta))} \\
& \times \sqrt{E V_{k}(x(t), t, r(t))}+I_{k}(t),
\end{aligned}
$$

for all $t \geq 0$. If $p_{k}-\sum_{l=1}^{n} q_{k l}>0$, then for any initial data $\xi \in C, r_{0} \in \mathcal{M}$, the solution of system (1) has the following property

$$
E V_{k}(x(t), t, r(t)) \leq K e^{-\lambda t}+\beta^{t}, \quad t \geq 0, k=1,2, \cdots, n
$$

where $K=\sum_{k=1}^{n} E V_{k}(x(0), 0, r(0)), \lambda=\min _{1 \leq k \leq n} \lambda_{k}, \lambda_{k}=\sup \left\{\varrho>0, p_{k}-\sum_{l=1}^{n} q_{k l} \varrho^{\varrho \tau}-\varrho>0\right\}, \beta^{t}=\max _{1 \leq k \leq n}\left(\left(p_{k}-\right.\right.$ $\left.\left.\sum_{l=1}^{n} q_{k l}\right)^{-1} I_{k}^{t}\right), I_{k}^{t}=\sup _{0 \leq s \leq t} I_{k}(s), I^{t}=\left(I_{1}^{t}, \cdots, I_{n}^{t}\right)^{T}$.

proof: Let us first prove that $\lambda>0$. In fact, for any fixed $k$, let $F_{k}(\varrho)=p_{k}-\sum_{l=1}^{n} q_{k l} \varrho^{\varrho \tau}-\varrho$. Obvious, $F_{k}(0)>0$. From $F_{k}(\infty)<0$ and $\dot{F}_{k}(\varrho)=-\tau \sum_{l=1}^{n} q_{k l} e^{\varrho \tau}-1<0$, there must exist a unique $\lambda_{k}>0$ such that $F_{k}\left(\lambda_{k}\right)=0$. From the definition of $\lambda_{k}$, we have $\lambda>0$. In order to prove (3), it is sufficient to prove $\forall \lambda^{*} \in(0, \lambda)$

$$
E V_{k}(x(t), t, r(t))-\beta^{t} \leq K e^{-\lambda^{*} t}, \quad t \geq 0 .
$$

Let $W_{k}(t)=E V_{k}(x(t), t, r(t))-\beta^{t}, Z(t)=K e^{-\lambda^{*} t}$ for simplicity. If (4) is not true, then there must be a constant $t^{*}>0$ and $\bar{k} \in\{1,2, \cdots, n\}$ such that

$$
\begin{gathered}
W_{\bar{k}}\left(t^{*}\right)=Z\left(t^{*}\right), \\
D^{+} W_{\bar{k}}\left(t^{*}\right) \geq D^{+} Z\left(t^{*}\right), \\
W_{k}(t) \leq Z(t), \quad k=1,2, \cdots, n, \quad t \in\left[0, t^{*}\right] .
\end{gathered}
$$

Since the $\beta_{k}^{t}$ is non-decreasing with $t$, simple computation shows that

$$
D^{+} W_{k}\left(t^{*}\right)=D^{+}\left(E V_{k}\left(x\left(t^{*}\right), t^{*}, r\left(t^{*}\right)\right)-\beta_{k}^{t}\right) \leq D^{+} E V_{k}\left(x\left(t^{*}\right), t^{*}, r\left(t^{*}\right)\right) .
$$

Together with condition (2) yields

$$
\begin{aligned}
D^{+} W_{\bar{k}}\left(t^{*}\right) \leq D^{+} E V_{\bar{k}}\left(x\left(t^{*}\right), t^{*}, r\left(t^{*}\right)\right) \\
\quad \leq-p_{\bar{k}} E V_{\bar{k}}\left(x\left(t^{*}\right), t^{*}, r\left(t^{*}\right)\right)+\sum_{l=1}^{n} q_{\bar{k} l} \sup _{-\tau \leq \theta \leq 0} \sqrt{E V_{l}\left(x\left(t^{*}+\theta\right), t^{*}+\theta, r\left(t^{*}+\theta\right)\right)} \sqrt{E V_{\bar{k}}\left(x\left(t^{*}\right), t^{*}, r\left(t^{*}\right)\right)}+I_{\bar{k}}^{t^{*}} \\
\quad \leq-p_{\bar{k}}\left(K \exp \left\{-\lambda^{*} t^{*}\right\}+\beta^{t}\right)+\sum_{l=1}^{n} q_{\bar{k} l} \sqrt{\left(K \exp \left\{-\lambda^{*} t^{*}\right\}+\beta^{t}\right)\left(K \exp \left\{-\lambda^{*}\left(t^{*}-\tau\right)\right\}+\beta^{t}\right)+I_{\bar{k}}^{t^{*}}} \\
\quad<-p_{\bar{k}}\left(K e^{-\lambda^{*} t^{*}}+\beta^{t}\right)+\sum_{l=1}^{n} q_{\bar{k} l}\left(K \exp \left\{-\lambda^{*}\left(t^{*}-\tau\right)\right\}+\beta^{t}\right)+I_{\bar{k}}^{t^{*}} \\
\quad<\left(\sum_{l=1}^{n} q_{\bar{k} l} e^{\lambda^{*} \tau}-p_{\bar{k}}\right) K e^{-\lambda^{*} t^{*}}-\left(p_{\bar{k}}-\sum_{l=1}^{n} q_{\bar{k} l}\right) \beta^{t}+I_{\bar{k}}^{t^{*}} .
\end{aligned}
$$


It follows from the definition of $\lambda$ and $\beta^{t^{*}}$ that

$$
p_{k}-\sum_{l=1}^{n} q_{k l} e^{\lambda_{k} \tau}-\lambda^{*}>0, I_{\bar{k}}^{t^{*}} \leq\left(p_{\bar{k}}-\sum_{l=1}^{n} q_{\bar{k} l}\right) \beta^{t^{*}} .
$$

Substituting (9) into (8) yields

$$
D^{+} W_{\bar{k}}\left(t^{*}\right)<-\lambda^{*} K \exp \left\{-\lambda^{*} t^{*}\right\}=D^{+} Z_{\bar{k}}\left(t^{*}\right)
$$

It contradicts the inequality (6). Hence, (4) holds for all $t \geq 0$. Therefore, letting $\lambda^{*} \rightarrow \lambda$, (2) holds. The proof is completed.

Remark 3.1: In comparison with the vector $\mathscr{L}$-operator differential inequality present in [5] and [8], the time-delayed item and non-delay item are mixed in the differential inequality (2). Thus the magnification on the cross-item by the elemental inequality could be avoided.

Remark 3.2: When $I_{k}(t)=0$, the inequalities (2) can be rewritten as:

$$
D^{+} E V_{k}(x(t), t, r(t)) \leq-p_{k} E V_{k}(x(t), t, r(t))+\sum_{l=1}^{n} q_{k l} \sup _{-\tau \leq \theta \leq 0} \sqrt{E V_{l}(x(t+\theta), t+\theta, r(t+\theta))} \sqrt{E V_{k}(x(t), t, r(t))} .
$$

It is just the Halanay inequalities that presented by Shen and Wang in [12] with constant coefficient. Hence, the vector Halanay inequalities have been extended to a more general form.

Now we will provide a improved condition for vector $\mathscr{L}$-operator differential inequality (10) on exponential stability by using the property of M-matrix.

Lemma 3.2: Assume that $x(t)$ is a real-valued $\mathcal{F}_{t}$-adapted process on $[-\tau, \infty)$, let $P=\operatorname{diag}\left\{p_{1}, \cdots, p_{n}\right\}, Q=\left(q_{k l}\right)_{n \times n}$ with $p_{k}>0, q_{k l} \geq 0, k, l=1,2, \cdots, n$. Moreover, there exist functions $V_{1}, \cdots, V_{n} \in C\left(R^{n} \times R_{+} \times \mathcal{M}, R_{+}\right)$such that (10) holds for all $t \geq 0$. If $P-Q$ is a nonsingular $M$-matrix, then for any initial data $\xi \in C, r_{0} \in \mathcal{M}$, the solution of system (1) has the following property

$$
\left(E V_{k}(x(t), t, r(t))\right)^{1 / 2} \leq \alpha_{k} K^{1 / 2} \exp \{-\lambda t\}, k=1,2, \cdots, n,
$$

$t \geq 0$, where $\alpha=\left(\alpha_{1}, \cdots, \alpha_{n}\right)^{T} \gg 0, \min _{1 \leq k \leq n} \alpha_{k} \geq 1, \lambda=\min _{1 \leq k \leq n} \sup \left\{\varrho>0,0.5 \alpha_{k} p_{k}-\sum_{l=1}^{n} 0.5 \alpha_{l} q_{k l} e^{\varrho \tau}-\alpha_{k} \varrho>0\right\}$, $K$ are defined in Lemma 3.1.

proof: Since $P-Q$ is a nonsingular M-matix, there must exist $\alpha=\left(\alpha_{1}, \cdots, \alpha_{n}\right)^{T} \gg 0$ with $\min _{1 \leq k \leq n} \alpha_{k} \geq 1$, such that $p_{k} \alpha_{k}-\sum_{l=1}^{n} \alpha_{l} q_{k l}>0$. By virtue of similar technique presented in the Lemma 3.1, we can show that $\lambda>0$. Now we process to prove (11). It is sufficient to prove $\forall \lambda^{*} \in(0, \lambda)$

$$
\left(E V_{k}(x(t), t, r(t))\right)^{1 / 2} \leq \alpha_{k} K^{1 / 2} e^{-\lambda^{*} t}, \quad t \geq 0 .
$$

Let $W_{k}(t)=E V_{k}(x(t), t, r(t)), H_{K}(t)=\alpha_{k} K^{1 / 2} e^{-\lambda^{*} t}$ for simplicity. If (12) is not true, then there must exist a constant $t^{*}>0$ and $\bar{k} \in\{1,2, \cdots, n\}$ such that

$$
\begin{gathered}
W_{\bar{k}}\left(t^{*}\right)=H_{k}\left(t^{*}\right), \\
D^{+} W_{\bar{k}}\left(t^{*}\right) \geq D^{+} H_{k}\left(t^{*}\right), \\
W_{k}(t) \leq H_{k}(t), \quad k=1,2, \cdots, n, \quad t \in\left[0, t^{*}\right] .
\end{gathered}
$$


By the definition of $\lambda$, we have $-\lambda^{*} \alpha_{k}+0.5 p_{k} \alpha_{k}+\sum_{j=1}^{n} 0.5 \alpha_{l} q_{k l} \exp \left\{\lambda^{*} \tau\right\}<0$. Simple computation shows that

$$
\begin{aligned}
D^{+} H_{\bar{k}}\left(t^{*}\right) & =-\lambda^{*} \alpha_{\bar{k}} K^{1 / 2} \exp \left\{-\lambda^{*} t^{*}\right\} \\
& >0.5\left(-p_{\bar{k}} \alpha_{\bar{k}}+\sum_{l=1}^{n} q_{\bar{k} l} \alpha_{l} \exp \left\{\lambda^{*} \tau\right\}\right) K^{1 / 2} \exp \left\{-\lambda^{*} t^{*}\right\} \\
& \geq-0.5 p_{\bar{k}} \alpha_{\bar{k}} K^{1 / 2} \exp \left\{-\lambda^{*} t^{*}\right\}+\sum_{l=1}^{n} q_{\bar{k} l} \alpha_{l} \exp \left\{\lambda^{*} \tau\right\} K^{1 / 2} \exp \left\{-\lambda^{*}\left(t^{*}-\tau\right)\right\} \\
& \geq-0.5 p_{\bar{k}} W_{\bar{k}}\left(t^{*}\right)+0.5 \sum_{l=1}^{n} q_{k l} \sup _{-\tau \theta \leq 0} W_{l}\left(t^{*}+\theta\right) .
\end{aligned}
$$

Nothing that

$$
2 W_{\bar{k}}\left(t^{*}\right) D^{+} W_{\bar{k}}\left(t^{*}\right) \leq-p_{\bar{k}} W_{\bar{k}}^{2}\left(t^{*}\right)+\sum_{l=1}^{n} q_{\bar{k} l} W_{\bar{k}}\left(t^{*}\right) \sup _{-\tau \theta \leq 0} W_{l}\left(t^{*}+\theta\right) .
$$

This implies

$$
D^{+} H_{\bar{k}}\left(t^{*}\right)>D^{+} W_{\bar{k}}\left(t^{*}\right) .
$$

It contradicts the inequality (15). Hence, (12) holds for all $t \geq 0$. Therefore, letting $\lambda^{*} \rightarrow \lambda,(11)$ holds. The proof is completed.

Remark 3.3: Obviously, the condition $p_{k}>\sum_{l=1}^{n} q_{k l}$ has been weakened to that $P-Q$ is nonsingular M-matrix. As a result, the condition presented in [12] has been improved.

\subsection{Exponential input-to-state stability}

The main aim of the subsection is to establish criterion on mean square exponential ISS by the generalized vector Halanay inequality presented in Lemma 3.1.

Theorem 3.1: Let Assumption 1 - 3 hold. If there exist positive diagonal matrices $S(i)=\operatorname{diag}\left\{s_{1}(i), \cdots, s_{n}(i)\right\}, i \in \mathcal{M}$, such that $p_{k}-\sum_{l=1}^{n} q_{k l}>0$, where

$$
\begin{aligned}
& p_{k}=\min _{i \in \mathcal{M}}\left\{\left(2 \underline{h}_{k} \delta_{k}(i)-\bar{h}_{k}-2 \bar{h}_{k} \bar{a}_{k k}(i) M_{k}\right)\left(s_{k}(i)\right)^{-2}-\sum_{j=1}^{N} \gamma_{i j}\left(s_{k}(j)\right)^{-2}\right\} \min _{i \in \mathcal{M}}\left\{s_{k}^{2}(i)\right\}, \\
& q_{k l}=\left\{\begin{array}{lc}
\max _{i \in \mathcal{M}}\left\{\left\{2 \bar{h}_{k}\left|b_{k k}(i)\right| M_{k}\left(s_{k}(i)\right)^{-2}\right\}\right\} \max _{i \in \mathcal{M}}\left\{s_{k}^{2}(i)\right\}, & k=l, \\
\max _{i \in \mathcal{M}}\left\{\left\{2 \bar{h}_{k}\left(s_{k}(i)\right)^{-2}\left(\left|a_{k l}(i)\right|+\left|b_{k l}(i)\right|\right) M_{l}\right\}\right\} \max _{i \in \mathcal{M}}\left\{s_{k}(i)\right\} \max _{i \in \mathcal{M}}\left\{s_{l}(i)\right\}, & k \neq l .
\end{array}\right.
\end{aligned}
$$

then the system (1) is mean-square exponentially input-to-state stable.

Proof: Let $x_{l}(t)=x\left(t, \xi, r_{0}\right), x_{l}^{\tau}(t)=\left(t-\tau_{l}(t), \xi, r_{0}\right)$ for simplicity. Applying the Itô formula [11] to $V_{k}(x(t), i)=$ $\left(s_{k}(i)\right)^{-2} x_{k}^{2}(t)$ yields

$$
\begin{aligned}
\mathscr{L} V_{k}\left(x(t), x^{\tau}(t), i\right)= & 2\left(s_{k}(i)\right)^{-2} x_{k}(t) h_{k}\left(x_{k}(t)\right)\left[-d_{k}\left(i, x_{k}(t)\right)+\sum_{l=1}^{n} a_{k l}(i) f_{l}\left(x_{l}(t)\right)+\sum_{l=1}^{n} b_{k l}(i) f_{l}\left(x_{l}^{\tau}(t)\right)+u_{k}(t)\right] \\
& +\sum_{j=1}^{N} \gamma_{i j}\left(s_{k}(j)\right)^{-2} x_{k}^{2}(t) \\
\leq & -2 \underline{h}_{k}\left(s_{k}(i)\right)^{-2} x_{k}(t) d_{k}\left(i, x_{k}(t)\right)+2 \bar{h}_{k}\left(s_{k}(i)\right)^{-2}\left|x_{k}(t) \| u_{k}(t)\right|+2 \bar{h}_{k}\left(s_{k}(i)\right)^{-2} \sum_{l=1}^{n} a_{k l}(i) x_{k}(t) f_{l}\left(x_{l}(t)\right)
\end{aligned}
$$




$$
\begin{aligned}
& +2 \bar{h}_{k}\left(s_{k}(i)\right)^{-2} \sum_{l=1}^{n} b_{k l}(i) x_{k}(t) f_{l}\left(x_{l}^{\tau}(t)\right)+\sum_{j=1}^{N} \gamma_{i j}\left(s_{k}(j)\right)^{-2} x_{k}^{2}(t) \\
\leq & -2 \underline{h}_{k}\left(s_{k}(i)\right)^{-2} \delta_{k}(i) x_{k}^{2}(t)+\bar{h}_{k}\left(s_{k}(i)\right)^{-2}\left(\varepsilon_{k} x_{k}^{2}(t)+\frac{u_{k}^{2}(t)}{\varepsilon_{k}}\right)+2 \bar{h}_{k}\left(s_{k}(i)\right)^{-2} \sum_{l=1}^{n} a_{k l}(i) x_{k}(t) f_{l}\left(x_{l}(t)\right) \\
& +2 \bar{h}_{k}\left(s_{k}(i)\right)^{-2} \sum_{l=1}^{n} b_{k l}(i) x_{k}(t) f_{l}\left(x_{l}^{\tau}(t)\right)+\sum_{j=1}^{N} \gamma_{i j}\left(s_{k}(j)\right)^{-2} x_{k}^{2}(t) \\
\leq & \left.-p_{k}(\varepsilon, i)\right) \min _{i \in \mathcal{M}}\left(s_{k}(i)\right) \max _{i \in \mathcal{M}}\left(s_{k}(i)\right)^{-2} x_{k}^{2}(t)+\varepsilon_{k}^{-1} \bar{h}_{k} \max _{i \in \mathcal{M}}\left(s_{k}(i)\right)^{-2} u_{k}^{2}(t) \\
& +2 \bar{h}_{k}\left(s_{k}(i)\right)^{-2} \sum_{l=1, l \neq k}^{n}\left|a_{k l}(i)\right| M_{l} \max _{i \in \mathcal{M}}\left(s_{k}(i)\right) \max _{i \in \mathcal{M}}\left(s_{l}(i)\right) \min _{i \in \mathcal{M}}\left(s_{k}(i)\right)^{-1}\left|x_{k}(t)\right| \min _{i \in \mathcal{M}}\left(s_{l}(i)\right)^{-1}\left|x_{l}(t)\right| \\
& +2 \bar{h}_{k}\left(s_{k}(i)\right)^{-2} \sum_{l=1}^{n}\left|b_{k l}(i)\right| M_{l} \max _{i \in \mathcal{M}}\left(s_{k}(i)\right) \max _{i \in \mathcal{M}}\left(s_{l}(i) \min _{i \in \mathcal{M}}\left(s_{k}(i)\right)^{-1}\left|x_{k}(t)\right| \min _{i \in \mathcal{M}}\left(s_{l}(i)\right)^{-1}\left|\left(x_{l}^{\tau}(t)\right)\right|\right.
\end{aligned}
$$

where $p_{k}(\varepsilon, i)=\left(2 \underline{h}_{k} \delta_{k}(i)-\bar{h}_{k} \varepsilon_{k}-2 \bar{h}_{k} \bar{a}_{k k}(i) M_{k}\left(s_{k}(i)\right)^{-2}-\sum_{j=1}^{N} \gamma_{i j}\left(s_{k}(j)\right)^{-2}\right)$. The well-known Hölder inequality yields that

$$
\begin{aligned}
E \mathscr{L} V_{k}\left(x(t), x^{\tau}(t), i\right) \leq & \left.-p_{k}(\varepsilon, i)\right) \min _{i \in \mathcal{M}}\left(s_{k}(i)\right) E\left(\max _{i \in \mathcal{M}} V_{k}(x(t), i)\right)+I_{k}(t) \\
& +2 \bar{h}_{k}\left(s_{k}(i)\right)^{-2} \sum_{l=1, l \neq k}^{n}\left|a_{k l}(i)\right| M_{l} \max _{i \in \mathcal{M}}\left(s_{k}(i)\right) \max _{i \in \mathcal{M}}\left(s_{l}(i)\right) \sqrt{E \min _{i \in \mathcal{M}} V_{k}(x(t), i)} \sqrt{E \min _{i \in \mathcal{M}} V_{l}(x(t), i)} \\
& +2 \bar{h}_{k}\left(s_{k}(i)\right)^{-2} \sum_{l=1}^{n}\left|b_{k l}(i)\right| M_{l} \max _{i \in \mathcal{M}}\left(s_{k}(i)\right) \max _{i \in \mathcal{M}}\left(s_{l}(i) \sqrt{E \min _{i \in \mathcal{M}} V_{k}(x(t), i)} \sqrt{E \min _{i \in \mathcal{M}} V_{l}\left(x^{\tau}(t), i\right)},\right.
\end{aligned}
$$

where $I_{k}(t)=\varepsilon_{k}^{-1} \bar{h}_{k} \max _{i \in \mathcal{M}}\left(s_{k}(i)\right)^{-2} u_{k}^{2}(t)$. According to the generalized Itô formula [11] and Definition 2, we have

$$
\begin{aligned}
D^{+} E V_{k}(x(t), r(t)) \leq & -p_{k}(\varepsilon) E\left(V_{k}(x(t), r(t))+\sum_{l=1}^{n} q_{k l} \sup _{-\tau \leq \theta \leq 0}\left(\sqrt{E\left(V_{k}(x(t), r(t))\right.}\right.\right. \\
& \left.\times \sqrt{E\left(V_{l}(x(t+\theta), r(t))\right.}\right)+I_{k}(t) .
\end{aligned}
$$

where $p_{k}(\varepsilon)=\min _{i \in \mathcal{M}} p_{k}(\varepsilon, i) \min _{i \in \mathcal{M}}\left(s_{k}(i)\right)$. Since $P=\operatorname{diag}\left(p_{1}, \ldots, p_{n}\right)$ and $Q=\left(q_{k l}\right)_{n \times n}$ are continuously dependent on its elements, we can claim that there exists $\varepsilon>0$ such that $p_{k}(\varepsilon)-\sum_{l=1}^{n} q_{k l}>0, k=1,2, \cdots, n$. It follows from Lemma 3.1, for any $k=1,2, \cdots, n$

$$
E V_{k}(x(t), r(t)) \leq K e^{-\lambda t}+\beta^{t}, \quad t \geq 0,
$$

where $\beta^{t}=\max _{1 \leq k \leq n}\left(\left(p_{k}(\varepsilon)-\sum_{l=1}^{n} q_{k l}\right)^{-1} I_{k}^{t}\right)$ and $\lambda=\min _{1 \leq k \leq n} \sup \left\{\varrho>0, p_{k}(\varepsilon)-\sum_{l=1}^{n} q_{k l} e^{\varrho \tau}-\varrho>0\right\}$. Simple computation shows that

$$
\begin{aligned}
& K=\sum_{k=1}^{n} E V_{k}(x(0), r(0)) \leq \underline{s}^{-2} \sum_{k=1}^{n} E\left|x_{k}(0)\right|^{2} \leq \underline{s}^{-2} \sup _{-\tau \leq \theta \leq 0} E\|\xi(\theta)\|^{2}, \\
& E\left|x_{k}(t)\right|^{2} \leq \bar{s}^{2}\left(K e^{-\lambda t}+\beta^{t}\right) .
\end{aligned}
$$

where $\bar{s}=\max _{k \in \mathcal{M}, 1 \leq i \leq n} s_{k}(i), \underline{s}=\min _{k \in \mathcal{M}, 1 \leq i \leq n} s_{k}(i)$. By the definition of $I_{k}^{t}$, we have

$$
\begin{aligned}
\beta^{t} & =\max _{1 \leq k \leq n}\left(\left(p_{k}(\varepsilon)-\sum_{l=1}^{n} q_{k l}\right)^{-1} I_{k}^{t}\right)=\varepsilon^{-1} \underline{s}^{-2} \max _{1 \leq k \leq n}\left(\left(p_{k}(\varepsilon)-\sum_{l=1}^{n} q_{k l}\right)^{-1} \bar{h}_{k}\right) \sup _{0 \leq s \leq t} \sum_{k=1}^{n} u_{k}^{2}(s) \\
& \leq \varepsilon^{-1} \underline{s}^{-2} \max _{1 \leq k \leq n}\left(\left(p_{k}(\varepsilon)-\sum_{l=1}^{n} q_{k l}\right)^{-1} \bar{h}_{k}\right)\|u\|_{\infty}^{2} .
\end{aligned}
$$


Substituting (18) and (20) into (19) yields

$$
E\left|x_{k}(t)\right|^{2} \leq \bar{s}^{2} \underline{s}^{2} E\|\xi\|_{\tau} e^{-\lambda t}+\max _{k \in \mathcal{M}}\left(\varepsilon_{k}^{-1} \bar{h}_{k}\right) \underline{s}^{-2}\|u\|_{\infty}^{2},
$$

Then we have

$$
E\|x(t)\|^{2} \leq L E\|\xi\|_{\tau} e^{-\lambda t}+\varphi\left(\|u\|_{\infty}^{2}\right),
$$

where $L=n \bar{s}^{2} \underline{s}^{2}, \varphi(t)=N \varepsilon^{-1} \underline{s}^{-2} \max _{1 \leq k \leq n}\left(\left(p_{k}(\varepsilon)-\sum_{l=1}^{n} q_{k l}\right)^{-1} \bar{h}_{k}\right) t$.

Hence, from Definition 1, the DCGNN with Markovian switching (1) is mean-square exponentially input-to-state stable. This completes the proof of Theorem 3.1.

Noting that when $h_{k}\left(x_{k}(t)\right)=1, d_{k}\left(i, x_{k}(t)\right)=d_{k}(i) x_{k}(t)$, system (1) degenerates into the following recurrent neural network presented in [9]

$$
d x(t)=[-D(r(t)) x(t)+A(r(t)) f(x(t))+B(r(t)) f(x(t-\tau(t)))+U(t)] d t .
$$

Then the parameters in Theorem 3.2 can be rewritten as

$$
\begin{aligned}
& p_{k}=\min _{i \in \mathcal{M}}\left\{\left(2 d_{k}(i)-2 \bar{a}_{k k}(i) M_{k}\right)\left(s_{k}(i)\right)^{-2}-\sum_{j=1}^{N} \gamma_{i j}\left(s_{k}(j)\right)^{-2}\right\}\left\{\min _{i \in \mathcal{M}} s_{k}^{2}(i)\right\}, \\
& q_{k l}= \begin{cases}\max _{i \in \mathcal{M}}\left(2\left(s_{k}(i)\right)^{-2}\left(\left|a_{k l}(i)\right|+\left|b_{k l}(i)\right|\right) M_{l}\right) \max _{i \in \mathcal{M}}\left\{s_{k}(i)\right\} \max _{i \in \mathcal{M}}\left\{s_{l}(i)\right\}, & k \neq l, \\
\max _{i \in \mathcal{M}}\left(2\left(s_{k}(i)\right)^{-2}\left|b_{k k}(i)\right| M_{k}\right) \max _{i \in \mathcal{M}}\left\{s_{k}^{2}(i)\right\}, & k=l .\end{cases}
\end{aligned}
$$

Then Theorem 3.1 has a concise form for system (21) as follows:

Corollary 3.1: System (21) is said to be mean-square exponentially input-to-state stable, if there exist positive diagonal matrices $S(i)=\operatorname{diag}\left\{s_{1}(i), \cdots, s_{n}(i)\right\}, i \in \mathcal{M}$, such that $p_{k}-\sum_{l=1}^{n} q_{k l}>0$.

Remark 3.4: Compared with the existing criteria on ISS in [5], [9] and [31], the amplification on cross items by the elemental inequality and Younger inequality could be avoided with the help of generalized vector Halanay inequality presented in Lemma 3.1. It also can be concluded that our ISS criterion has less conservative than the existing criteria in [5], [9] and [31], which can be illustrated by the numerical Example 4.1.

\subsection{Exponential stability}

When $U(t) \equiv 0$, in this case, the exponential ISS degenerate into the exponential stability. In this subsection, we assume that (1) has a unique equilibrium point $x^{*}$. Let $y(t)=x(t)-x^{*}, g(y(\cdot))=f\left(x(\cdot)+x^{*}\right)-f\left(x^{*}\right)$. Then (1) can be rewritten as

$$
d y(t)=H(y(t))[-D(r(t), y(t))+A(r(t)) g(y(t))+B(r(t)) g(y(t-\tau(t)))] d t .
$$

In addition, the activation function $g$ in (24) is also monotone nondecreasing and Lipschitz continuous like $f$ in Assumption 3 .

Theorem 3.2: Assume that there exist positive diagonal matrices $S(i)=\operatorname{diag}\left\{s_{1}(i), \cdots, s_{n}(i)\right\}, i \in \mathcal{M}$, such that $P-Q$ is a non-singular M-matrix with $P=\operatorname{diag}\left(p_{1}, \cdots, p_{n}\right), Q=\left(q_{k l}\right)_{n \times n}$, then for any initial value $\xi \in C, r_{0} \in \mathcal{M}$, the state $y\left(t, \xi, r_{0}\right)$ of (24) satisfies

$$
\limsup _{t \rightarrow \infty} \frac{\ln E\left|y\left(t, \xi, r_{0}\right)\right|^{2}}{t} \leq-2 \lambda, \quad \limsup _{t \rightarrow \infty} \frac{\ln \left|y\left(t, \xi, r_{0}\right)\right|}{t} \leq-\lambda, \text { a.s. }
$$


i.e., (24) is almost surely exponentially stable and mean square exponentially stable, where $\lambda=\min _{1 \leq k \leq n} \sup \{\varrho>$ $\left.0,0.5 \alpha_{k} p_{k}-\sum_{l=1}^{n} 0.5 \alpha_{l} q_{k l} e^{\varrho \tau}-\alpha_{k} \varrho>0\right\}$, with $\alpha=\left(\alpha_{1}, \cdots, \alpha_{n}\right)^{T} \gg 0$ such that $(P-Q) \alpha \gg 0, \min _{1 \leq k \leq n} \alpha_{k} \geq 1$, and $p_{k}, q_{k l}$ are defined in Theorem 3.1.

Proof: Let $y_{l}(t)=y_{l}\left(t, \xi, r_{0}\right), y_{l}^{\tau}(t)=y_{l}\left(t-\tau_{l}(t), \xi, r_{0}\right)$ for simplicity. Applying the Itô formula to $V_{k}(y(t), i)=$ $\left(s_{k}(i)\right)^{-2}\left|y_{k}(t)\right|^{2}$ yields

$$
\begin{aligned}
\mathscr{L} V_{k}\left(y(t), y^{\tau}(t), i\right) \leq & -2 \underline{h}_{k}\left(s_{k}(i)\right)^{-2} y_{k}(t) d_{k}\left(i, y_{k}(t)\right)+2 \bar{h}_{k}\left(s_{k}(i)\right)^{-2}\left|y_{k}(t)\right|\left|u_{k}(t)\right|+2 \bar{h}_{k}\left(s_{k}(i)\right)^{-2} \sum_{l=1}^{n} a_{k l}(i) y_{k}(t) f_{l}\left(y_{l}(t)\right) \\
& +2 \bar{h}_{k}\left(s_{k}(i)\right)^{-2} \sum_{l=1}^{n} b_{k l}(i) y_{k}(t) f_{l}\left(y_{l}^{\tau}(t)\right)+\sum_{j=1}^{N} \gamma_{i j}\left(s_{k}(j)\right)^{-2} y_{k}^{2}(t) \\
\leq & -\left[2 \underline{h}_{k}\left(s_{k}(i)\right)^{-2} \delta_{k}(i)-2\left(s_{k}(i)\right)^{-2} \bar{h}_{k} \bar{a}_{k k}(i) M_{k}-\sum_{j=1}^{N} \gamma_{i j}\left(s_{k}(j)\right)^{-2}\right] y_{k}^{2}(t) \\
& +2 \bar{h}_{k}\left(s_{k}(i)\right)^{-2} \sum_{l=1, l \neq k}^{n}\left|a_{k l}(i)\right| M_{l}\left|y_{k}(t)\right|\left|y_{l}(t)\right|+2 \bar{h}_{k}\left(s_{k}(i)\right)^{-2} \sum_{l=1}^{n}\left|b_{k l}(i)\right| M_{l}\left|y_{k}(t)\right|\left|y_{l}^{\tau}(t)\right| \\
\leq & -\left[2 \underline{h}_{k}\left(s_{k}(i)\right)^{-2} \delta_{k}(i)-2\left(s_{k}(i)\right)^{-2} \bar{h}_{k} \bar{a}_{k k}(i) M_{k}-\sum_{j=1}^{N} \gamma_{i j}\left(s_{k}(j)\right)^{-2}\right] \min _{i \in \mathcal{M}}\left(s_{k}(i)\right) \max _{i \in \mathcal{M}}\left(s_{k}(i)\right)^{-2} y_{k}^{2}(t) \\
& +2 \bar{h}_{k}\left(s_{k}(i)\right)^{-2} \sum_{l=1, l \neq k}^{n}\left|a_{k l}(i)\right| M_{l} \max _{i \in \mathcal{M}}\left(s_{k}(i)\right) \max _{i \in \mathcal{M}}\left(s_{l}(i)\right) \min _{i \in \mathcal{M}}\left(s_{k}(i)\right)^{-1}\left|y_{k}(t)\right| \min _{i \in \mathcal{M}}\left(s_{l}(i)\right)^{-1}\left|y_{l}(t)\right| \\
& +2 \bar{h}_{k}\left(s_{k}(i)\right)^{-2} \sum_{l=1}^{n}\left|b_{k l}(i)\right| M_{l} \max _{i \in \mathcal{M}}\left(s_{k}(i)\right) \max _{i \in \mathcal{M}}\left(s_{l}(i) \min _{i \in \mathcal{M}}\left(s_{k}(i)\right)^{-1}\left|y_{k}(t)\right| \min _{i \in \mathcal{M}}\left(s_{l}(i)\right)^{-1}\left|\left(y_{l}^{\tau}(t)\right)\right|\right.
\end{aligned}
$$

The well-known Hölder inequality yields that

$$
\begin{aligned}
E \mathscr{L} V_{k}\left(y(t), y^{\tau}(t), i\right) \leq & -\left[2 \underline{h}_{k}\left(s_{k}(i)\right)^{-2} \delta_{k}(i)-2\left(s_{k}(i)\right)^{-2} \bar{h}_{k} \bar{a}_{k k}(i) M_{k}-\sum_{j=1}^{N} \gamma_{i j}\left(s_{k}(j)\right)^{-2}\right] \min _{i \in \mathcal{M}}\left(s_{k}(i)\right) E\left(\max _{i \in \mathcal{M}} V_{k}(y(t), i)\right) \\
& +2 \bar{h}_{k}\left(s_{k}(i)\right)^{-2} \sum_{l=1, l \neq k}^{n}\left|a_{k l}(i)\right| M_{l} \max _{i \in \mathcal{M}}\left(s_{k}(i)\right) \max _{i \in \mathcal{M}}\left(s_{l}(i)\right) \sqrt{E \min _{i \in \mathcal{M}} V_{k}(y(t), i)} \sqrt{E \min _{i \in \mathcal{M}} V_{l}(y(t), i)} \\
& +2 \bar{h}_{k}\left(s_{k}(i)\right)^{-2} \sum_{l=1}^{n}\left|b_{k l}(i)\right| M_{l} \max _{i \in \mathcal{M}}\left(s_{k}(i)\right) \max _{i \in \mathcal{M}}\left(s_{l}(i) \sqrt{E \min _{i \in \mathcal{M}} V_{k}(y(t), i)} \sqrt{E \min _{i \in \mathcal{M}} V_{l}\left(y^{\tau}(t), i\right)} .\right.
\end{aligned}
$$

According to the generalized Itô formula [11] and Definition 2, we have

$$
\begin{aligned}
D^{+} E V_{k}(y(t), r(t)) \leq & -p_{k} E\left(V_{k}(y(t), r(t))+\sum_{l=1}^{n} q_{k l} \sup _{-\tau \leq \theta \leq 0}\left(\sqrt{E\left(V_{k}(y(t), r(t))\right.}\right.\right. \\
& \left.\times \sqrt{E\left(V_{l}(y(t+\theta), r(t))\right.}\right)+I_{k}(t) .
\end{aligned}
$$

By Lemma 3.2 we have

$$
E V_{k}(y(t), r(t)) \leq \alpha_{k}^{2} K e^{-2 \lambda t}, \quad t \geq 0 .
$$

Combining the definition of $V_{k}$, it implies

$$
\limsup _{t \rightarrow \infty} \frac{\ln E|y(t)|^{2}}{t} \leq-2 \lambda .
$$


i.e., (24) is exponential stability in mean square. Based on the mean square exponential stability and Assumption 3, the almost surely exponentially stable follows by Theorem 7 in [11]. Therefore, (25) holds. This completes the proof of Theorem 3.2.

Remark 3.5:Noting, for $h_{k}\left(y_{k}(t)\right)=1, d_{k}\left(i, y_{k}(t)\right)=d_{k}(i) y_{k}(t)$, system (24) reduces to RNNs with Markovian switching (see [12]). In [12], Shen and Wang showed that the RNNs with Markovian switching is exponentially stable under the condition $p_{k}-\sum_{l=1}^{n} q_{k l}>0$. Obviously, the $p_{k}-\sum_{l=1}^{n} q_{k l}>0$ implies that $P-Q$ is a nonsingular M-matrix when taking the same matrix $S(i), i \in \mathcal{M}$, and the convergence cannot be ascertained. As a result, the conditions imposed on exponential stability have been improved.

When $D(i, x)=D(x), A(i)=A, B(i)=B$, then system (24) becomes a deterministic CGNN

$$
\dot{x}_{k}(t)=h_{k}\left(y_{k}(t)\right)\left[-d_{k}\left(y_{k}(t)\right)+\sum_{l=1}^{n} a_{k l} f_{l}\left(y_{l}(t)\right)+\sum_{l=1}^{n} b_{k l} f_{l}\left(x_{l}\left(t-\tau_{l}(t)\right)\right)\right] .
$$

Remark 3.6: For system (27), the parameters in Theorem 3.2 become

$$
p_{k}=\underline{h}_{k} \delta_{k}-\bar{h}_{k} \bar{a}_{k k} M_{k}, \quad q_{k l}= \begin{cases}\bar{h}_{k}\left|b_{k k}\right| M_{k}, & k=l, \\ \bar{h}_{k} \mid\left(a_{k l}|+| b_{k l} \mid\right) M_{l} \cdot\left(s_{k}\right)^{-1} \cdot s_{l}, & k \neq l .\end{cases}
$$

and the main condition becomes $\operatorname{diag}\left\{\delta_{1}, \cdots, \delta_{n}\right\}-S^{-1}(|\bar{A}|+|B|) M S$ is a non-singular M-matrix. Noting that

$$
S^{-1}\left(\operatorname{diag}\left\{\delta_{1}, \cdots, \delta_{n}\right\}-(|\bar{A}|+|B|) M\right) S=\operatorname{diag}\left\{\delta_{1}, \cdots, \delta_{n}\right\}-S^{-1}(|\bar{A}|+|B|) M S,
$$

we then obtain the following corollary.

Corollary 3.2: If $\operatorname{diag}\left\{\delta_{1}, \cdots, \delta_{n}\right\}-(|\bar{A}|+|B|) M$ is a non-singular M-matrix, then the deterministic neural network (27) is almost surely exponentially stable.

Remark 3.7: Noting that when $h_{k}\left(y_{k}(t)\right)=1, d_{k}\left(y_{k}(t)\right)=d_{k} y_{k}(t)$, system (27) reduces to the following RNN

$$
\dot{x}(t)=[-D y(t)+A f(y(t))+B f(y(t-\tau(t)))] .
$$

The criterion becomes that $(D-(|\bar{A}|+|B|) M)$ is a non-singular M-matrix. This happens to be the main results in [32]-[34]. Hence, Theorem 3.3 and Corollary 3.2 developed here are more general than those in the above mentioned literatures.

\section{Numerical examples}

In this section, two examples are demonstrated to show the efficiency of the criteria derived in Section 3.

Example 4.1: Consider the following two-neuron neural network with Markovian switching:

$$
\dot{x}(t)=H(x(t))[-D(i, x(t))+A(i) f(x(t))+B(i) f(x(t-\tau(t)))+U(t)],
$$

where the generator of the Markovian chain and parameters are

$$
\begin{gathered}
\Gamma=\left(\begin{array}{cc}
-1 & 1 \\
0.5 & -0.5
\end{array}\right), \quad D(1)=\left(\begin{array}{ll}
1 & 0 \\
0 & 2
\end{array}\right), \quad D(2)=\left(\begin{array}{cc}
1 & 0 \\
0 & 1.5
\end{array}\right), \quad A(1)=\left(\begin{array}{cc}
-0.8 & 0.1 \\
0.4 & -1.3
\end{array}\right), \\
A(2)=\left(\begin{array}{cc}
-0.5 & 0 \\
-0.2 & -2
\end{array}\right), B(1)=\left(\begin{array}{cc}
-0.1 & 0.1 \\
-0.2 & 0
\end{array}\right), \quad B(2)=\left(\begin{array}{cc}
0 & 0.2 \\
-0.3 & 0
\end{array}\right), \quad U(t)=\left(\begin{array}{c}
0.1 \sin t \\
0.1 \cos t
\end{array}\right),
\end{gathered}
$$




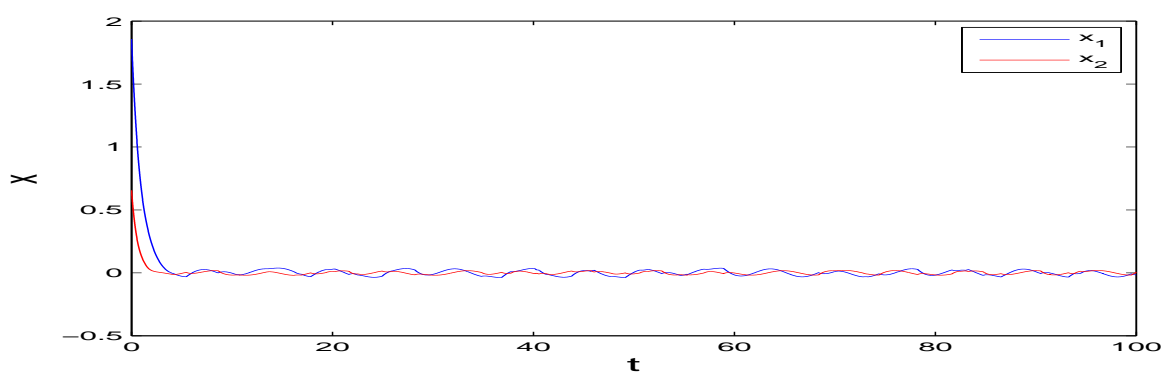

(a)

Figure(a). The transient state of $x_{1}(t), x_{2}(t)$ in present of input $u(t)$.

and $h_{1}\left(x_{1}(t)\right)=0.9+0.1 \sin x_{1}(t), h_{2}\left(x_{2}(t)\right)=0.7+0.1 \cos x_{2}(t), f_{i}(x)=0.2 \tanh (x(i)), i=1,2$. Taking $\varepsilon_{1}(1)=0.4$, $\varepsilon_{1}(2)=0.4, \varepsilon_{2}(1)=0.5, \varepsilon_{2}(2)=0.2, S(1)=\operatorname{diag}\{1,2\}, S(2)=\operatorname{diag}\{1,2\}, M_{1}=M_{2}=1$, a simple computation yields

$$
p_{1}=1.2, p_{2}=1.64, q_{11}=0.2, q_{12}=0.8, q_{21}=0.48, q_{22}=0 .
$$

Clearly, all the conditions of Theorem 3.1 are satisfied. Therefore, from Theorem 3.1, we can see that the DCGNNs (29) are exponentially input-to-state stable.

Remark 4.1: When the state space $\mathcal{M}=\{1\}$, the system (1) degenerates into a deterministic system in [5] with $\sigma_{i j}=0$ and $c_{i j}=0$

$$
\dot{x}(t)=H(x(t))[-D(1, x(t))+A(1) f(x(t))+B(1) f(x(t-\tau(t)))+U(t))] .
$$

Then, by Theorem 3.1, we can see that the system (30) is exponentially input-to-state stable. Now we process to apply the criteria in [5] to show the effectiveness of our results. For system (30), the parameters in [5] can be rewritten as

$$
T_{11}=0.5, T_{22}=0.5, T_{12}=0.05, T_{21}(t)=0.8, H_{11}=0.1, H_{12}=0.05, H_{21}=0.4, H_{22}=0 .
$$

It is easy to see the condition $\sum_{j=1}^{2}\left[T_{1 j}(t)+H_{1 j}\right]=0.5+0.05+0.1+0.05=0.7<0$ of the criteria is not satisfied. It implies that the system (30) can not be easily tackled by the method in [5].

Example 4.2: When $H(x(t))=1, D(i, x(t))=D(i) x(t)$, consider the following two-neuron neural networks with Markovian switching:

$$
\dot{x}(t)=-D(r(t)) x(t)+A(r(t)) f(x(t))+B(r(t)) f(x(t-\tau(t))),
$$

where the generator of Markov chain and parameters are

$$
\begin{gathered}
\Gamma=\left(\begin{array}{cc}
-1 & 1 \\
\frac{1}{2} & -\frac{1}{2}
\end{array}\right), \quad D(1)=\left(\begin{array}{cc}
1 & 0 \\
0 & 1
\end{array}\right), \quad D(2)=\left(\begin{array}{ll}
1 & 0 \\
0 & 1
\end{array}\right), \quad A(1)=\left(\begin{array}{cc}
1.2 & 0.8 \\
0.8 & 0
\end{array}\right), \\
A(2)=\left(\begin{array}{cc}
-1 & 0.1 \\
0 & -0.3
\end{array}\right), \quad B(1)=\left(\begin{array}{cc}
1 & 0.9 \\
0.9 & 0.7
\end{array}\right), \quad B(2)=\left(\begin{array}{ll}
0.2 & 0.3 \\
0.3 & 0.5
\end{array}\right) .
\end{gathered}
$$

and $f(x)=0.15 x$. Taking $M_{1}=M_{2}=0.15, S(1)=\operatorname{diag}\{1.1,1.1\}, S(2)=\operatorname{diag}\{0.9,1\}$, the parameters in Theorem 3.2 can be easily derived as

$$
P=\left(\begin{array}{cc}
0.82 & 0 \\
0 & 0.9
\end{array}\right), \quad Q=\left(\begin{array}{cc}
0.3 & 0.26 \\
0.26 & 0.1
\end{array}\right)
$$

It is clear that $P-Q$ is a non-singular M-matrix. Therefore, from Theorem 3.3, we see that the system (31) is exponentially stable.

Remark 4.2: When choosing the same matrix $S(1)$ and $S(2)$ as that in Example 4.2, the parameters in [12] becomes

$$
p_{1}=0.77, p_{2}=1.47, q_{11}=0.3, q_{12}=0.5, q_{21}=0.51, q_{22}=0.21 .
$$

It is easy to verify that the conditions $p_{1}=0.77<\sum_{l=1}^{2} q_{1 l}=0.3+0.5=0.8$ of Theorem 4 in [12] do not hold. By Remark 3.5, we can conclude that the conditions of Theorem 3.2 are easier to verify than those in Theorem 4 in [12]. 


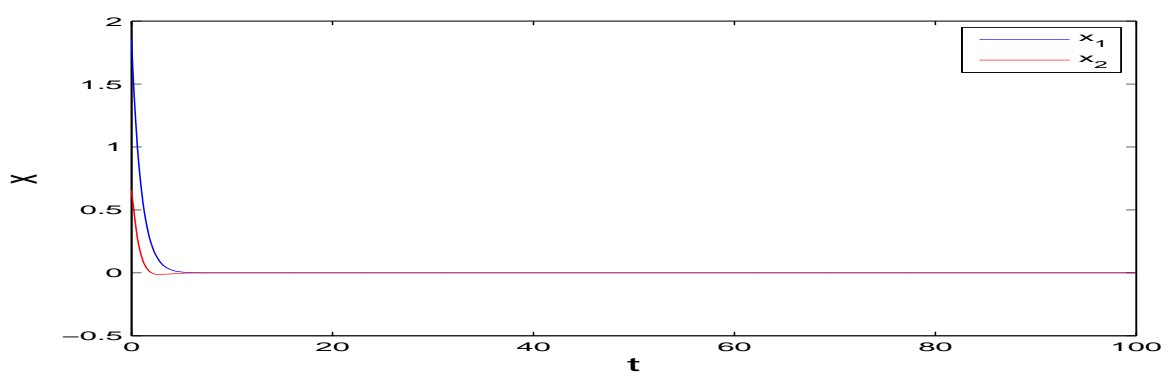

(b)

Figure(d). The transient state of $x_{1}(t), x_{2}(t)$ without input $u(t)$.

\section{Conclusions}

In this paper, we have investigated the stability for DCGNNs with Markovian switching. Firstly, by utilizing the vector Lyapunov function and property of M-matrix, two generalized Halanay inequalities have been established. Secondly, by applying the novel Halanay inequalities, sufficient criteria on mean-square exponential ISS for DCGNNs with Markovian switching have been obtained. Finally, two numerical examples and their simulations are provided to illustrate the effectiveness of our results.

\section{Acknowledgement}

The work was jointly supported by the National Science Foundation of China (Grant Nos. 61304070, 11271146, 61374080), the National Key Basic Research Program of China (973 Program) (2013CB228204), the Alexander von Humboldt Foundation of Germany (Fellowship CHN/1163390), the project funded by the Priority Academic Program Development of Jiangsu Higher Education Institutions and the Fundamental Research Funds for the Central Universities of China. (Grant No.2015B19814).

\section{References}

[1] M. Cohen, S. Grossberg. (1983). Absolute stability of global pattern formulation and parallel memory storage by competitive neural networks, IEEE Trans. Syst. Man Cybern. 13(5), 815-826.

[2] J. Cao, J. Liang. (2004). Boundedness and stability for Cohen-Grossberg neural network with time-varying delays, J. Math. Anal. Appl. 296(2), 665-685.

[3] Q. Song, J. Cao. (2006). Stability analysis of Cohen-Grossberg neural network with both time-varying and continuously distributed delays, J. Comput. Appl. Math. 197(1), 188-203.

[4] K. Yuan, J. Cao, H. Li. (2006). Robust stability of switched Cohen-Grossberg neural networks with mixed time-varying delays, IEEE Trans. Syst. Man Cybern. 36(6), 1356-1363.

[5] W. Zhou, L. Teng, D. Xu. (2015). Mean-square exponentially input-to-state stability of stochastic Cohen-Grossberg neural networks with time-varying delays, Neurocomputing 153, 54-61.

[6] Q. Zhu, X. Li. (2012). Exponential and almost sure exponential stability of stochastic fuzzy delayed Cohen-Grossberg neural networks, Fuzzy Set. Syst. 203(2), 74-94.

[7] Q. Zhu, J. Cao, R. Rakkiyappan. (2015). Exponential input-to-state stability of stochastic Cohen-Grossberg neural networks with mixed delays, Nonlinear Dyn. 79(2), 1085-1098.

[8] S. Long, D. Xu. (2013). Global exponential stability of non-autonomous cellular neural networks with impulses and time-varying delays, Commun. Nonlinear Sci. Numer. Simul. 18(6), 1463-1472.

[9] Y. Xu, W. Luo, K. Zhong, S. Zhu. (2014). Mean square input-to-state stability of a general class of stochastic recurrent neural networks with Markovian switching, Neural Comput Applic. 25(7), 1657-1663.

[10] H. Huang, D. Ho, Y. Qu. (2007). Robust stability of stochastic delayed additive neural networks with Markovian switching, Neural Netw. 20(7), 799-809.

[11] X. Mao, C. Yuan. (2006). Stochastic differential equations with Markovian switching, London,U.K.: Imperial College Press.

[12] Y. Shen, J.Wang. (2009). Almost sure exponential stability of recurrent neural networks with Markovian switching, IEEE Trans. Neural Netw. 20(5), 840-855.

[13] Q. Zhu, J. Cao. (2009). Stability analysis of Markovian jump stochastic BAM neural networks with impulse control and mixed time delays, IEEE Trans. Neural Netw. 23(3), 467-479. 
[14] Q. Zhu, J. Cao. (2010). Robust exponential stability of Markovian jump impulsive stochastic Cohen-Grossberg neural networks with mixed time delays, IEEE Trans. Neural Netw. 21(8), 1314-1325.

[15] Q. Zhu, J. Cao. (2011). Exponential stability of stochastic neural networks with both Markovian jump parameters and mixed time delays, IEEE Trans. Syst. Man Cybern.B 41(2), 341-353.

[16] L. Liu, Y. Shen, F. Jiang. (2011). The almost sure asymptotic stability and pth moment asymptotic stability of nonlinear stochastic differential systems with polynomial growth, IEEE Trans. Autom. Control 56(8), 1985-1990.

[17] L. Liu. (2016). New criteria on Exponential Stability for Stochastic Delay Differential Systems Based on Vector Lyapunov Function, IEEE Trans. Syst., Man, Cybern., Syst., doi: 10.1109/TSMC.2016.2558047.

[18] Y. Shen, J. Wang. (2008). An improved algebraic criterion for global exponential stability of recurrent neural networks with time-varying delays, IEEE Trans. Neural Netw. 19(3), 528-531.

[19] L. Wang, Y. Shen, Z. Ding. (2015). Finite time stabilization of delayed neural networks, Neural Netw. 70(C), 74-80.

[20] J. Xiao, Z. Zeng, A.Wu. (2014). New criteria for exponential stability of delayed recurrent neural networks, Neurocomputing 134(9), 182-188.

[21] G. Zhang, Y. Shen. (2015). Novel conditions on exponential stability of a class of delayed neural networks with state-dependent switching, Neural Netw. 71(C), 55-61.

[22] S. Zhu, K. Zhong, Y. Shen. (2014). Robustness analysis for parameter matrices of global exponential stability time varying delay systems, Appl. Math. Comput. 237, 386-395.

[23] E. Sanchez, J. Perez. (1999). Input-to-state stability analysis for dynamical neural networks, IEEE Trans. Circuits Syst. 46(11), 1395-1398.

[24] C. Ahn. (2011). Robust stability of recurrent neural networks with input-to-state stable learning algorithm, Nonlinear Dyn. 65(4), 413-419.

[25] Q. Zhu, J. Cao. (2014). Mean-square exponential input-to-state stability of stochastic delayed neural networks, Neurocomputing 131(9), 157-163.

[26] R. Bellman. (1962). Vector Lyapunov functions, SIAM J.Control. 1(1), 32-34.

[27] V. Matrosov. (1972). Method of vector Lyapunov functions of interconnected systems with distributed parameters(survey), Avtomat. i Telemekh. 33, 63-75.

[28] A. Berman, R. Plemmons. (1979). Nonnegative matrices in the mathematical sciences, New York: Academic.

[29] S. Berberian. (1999). Fundamentals of real analysis, New York: Springer.

[30] A. Halanay. (1966). Differential equations: stability, oscillation, timelags, New York: Academic.

[31] S. Zhu, Y. Shen. (2013). Two algebtaic criteria for input-to-state stability of recurrent neural networks with time-varying delays, Neurocomputing 22(6), 1163-1169.

[32] J. Cao, J. Wang. (2003). Global asymptotic stability of a general class of recurrent neural networks with time-varying delays, IEEE Trans. Circuits Syst. 50(1), 34-44.

[33] C. Feng, R. Plamondon. (2001). On the stability of delayed Hopfield neural networks, Neural Netw. 14, 1181-1188.

[34] P. Liu, Q. Han. (2006). On stability of recurrent neural networks:an approach from Volterra integro-differential equations, IEEE Trans. Neural Netw. 17(1), 264-267. 